\title{
A novel method for the construction of verbal rating scales at the ratio scale level of measurement precision: The multidimensional- psychophysical scaling procedure (M-PSP)
}

\author{
W. CRawFord Clark \\ Columbia University, New York, New York \\ and New York State Psychiatric Institute, New York, New York \\ J. Douglas Carroll \\ Rutgers University, Newark, New Jersey \\ AND \\ Malvin N. Janai \\ New York University, New York, New York
}

\begin{abstract}
The multidimensional-psychophysical scaling procedure was employed to construct verbal rating scales at the ratio scale level of measurement precision. The multidimensional individual differences scaling model (INDSCAL) demonstrated a one-dimensional solution in the group stimulus space, with the eight verbal descriptors and the eight electrical stimulus intensities interdigitated with respect to their perceived strength. A plot of these stimulus coordinates against a scale of electrical intensities in milliwatts yielded an exponential function. To construct the rating scales, the four attributes that could be discerned within the dimension - namely, perceived intensities of the electrical stimuli and the attributes of the subjective experiences evoked by the electrical stimuli: pain qualities, somatosensory qualities, and emotional qualities - were plotted as separate scales against a subjective intensity scale. Although not presented here, INDSCAL also yields a subject coordinate space that quantifies the importance or saliency to each subject of each pole, stoical or complainer, of the dimension.
\end{abstract}

The present article demonstrates a novel method for the construction of verbal rating scales. This method, the multidimensional-psychophysical scaling procedure (M-PSP), may be defined as a procedure for generating rating scales that quantify judgments of the perceived strength of physical stimuli and/or verbal descriptors at the ratio scale level of measurement precision. Unlike ordinal scales, ratio scales have a true zero point (e.g., 0 in. on a ruler) and permit the legitimate use of parametric statistics.

Rating scales are widely used in various fields to assess an individual's sensory sensitivity, emotional state, and preferences. Medically, patients' ratings of sensory and emotional experiences, such as pain, anxiety and depression, are important for diagnosis, selection of the optimal treatment strategy, and the evaluation of treatment outcome. Baird and Noma (1978) reviewed the history of the many attempts that have been made over the past centuries to determine valid procedures that relate physical quantities to a person's subjective perception of the strength or intensity of these quantities. Bernoulli (1738/1954) related actual economic values to their perceived value.
Fechner (1860/1966) and Stevens (1957) related sensory intensity to perceived sensory strength, whereas Gracely, McGrath, and Dubner (1978) related verbal descriptors of pain and emotion to their perceived strength.

The M-PSP method introduced here combines traditional threshold and multidimensional scaling procedures. Each of these procedures possesses advantages and disadvantages. The method of serial exploration (or limits) threshold has the advantage that it is directly related to readily understood measures of physical intensity, such as watts, lumens, decibels, grams, and so on. However, the threshold is subject to the influence of nonsensory variables, such as the subject's emotional state, sex, and ethnoculturally based attitudes (Clark, 2003b, 2003c). The multidimensional scaling model has the advantage that it reveals the number and characteristics of sensory and emotional dimensions in the subject's rather than the investigator's perceptual and cognitive worlds, including dimensions that the investigator may not have even envisioned. However, its disadvantage is that these dimensions, unlike the threshold measure, cannot be related to

W. C. Clark, clarker@pi.cpmc.columbia.edu 
physical units of intensity. The M-PSP combines the advantages of these two methods.

Various metric and nonmetric mulitidimensional scaling procedures were described by Lattin, Carroll, and Green (2003). One of their key ideas was that subjective similarity judgments made between pairs of stimulus objects, such as physical stimuli or words, that represent psychological distance can be used to scale physical distance. Multidimensional scaling models generate a configuration of points, stimulus coordinates, on one or more dimensions in the group stimulus space, as on a map. Of particular interest here is the individual differences scaling model (INDSCAL) conceived by Carroll and Chang (1970). In addition to yielding the group stimulus space that is common to all individuals, INDSCAL yields the source or subject coordinate space. The coordinates of this space identify the relative importance, or saliency, of each dimension to each subject or, in the instance of a one-dimensional solution, the relative importance of each pole of the dimension to each subject.

In the present study, we employed the results of an earlier study in which the stimulus objects consisted of both words and physical stimuli. Previously, like all other investigators, we had employed INDSCAL to determine group stimulus spaces that contained only verbal stimulus objects, such as descriptors of pain and emotion (Clark, Ferrer-Brechner, Janal, Carroll, \& Yang, 1989; Clark, Janal, Hoben, \& Carroll, 2001), or only sensory stimulus objects, such as heat (Clark, Carroll, Yang, \& Janal, 1986) or electrical stimuli (Janal, Clark, \& Carroll, 1993). Our experience with both types of stimulus objects led to the study in which both verbal and sensory stimulus objects were included (Janal, 1995). The present study was conceived some years later, when the early study was being reexamined by the senior author (Clark, 2003c).

\section{Methods and Results of the Earlier Study}

In Janal (1995), the 25 male $^{1}$ subjects made pairwise similarity ratings on a 10 -point numerical rating scale $(0$, not similar; 9 , extremely similar $)$.

The eight verbal stimulus objects were descriptors of perceptual qualities that ranged from slight sensation and tingling to severe pain and hammering, as well as descriptors of positive and negative emotions that ranged from comforting to upsetting. The eight sensory stimulus objects were 1.0 -sec trains of $1.0-\mathrm{msec}$ square-wave electrical pulses at a frequency of $10 \mathrm{~Hz}$ that ranged in intensity from 3.2 to $235 \mathrm{~mW}$. These stimulus objects appear in Figure 1.

The electrical stimuli were generated by a computercontrolled constant-current stimulator. The pulses were delivered to ring electrodes attached to the first and second phalanges of the index and middle fingers of the left hand. The device measured both the voltage and the current of each stimulus; this allowed the power, expressed in milliwatts, to be determined. Measures of power are desirable, because they provide comparable measures of stimulus intensity across subjects with different skin impedance.

The INDSCAL model (Carroll \& Chang, 1970) was employed to collect and analyze the data. The subjects
Stimuli (mW)

Descriptors

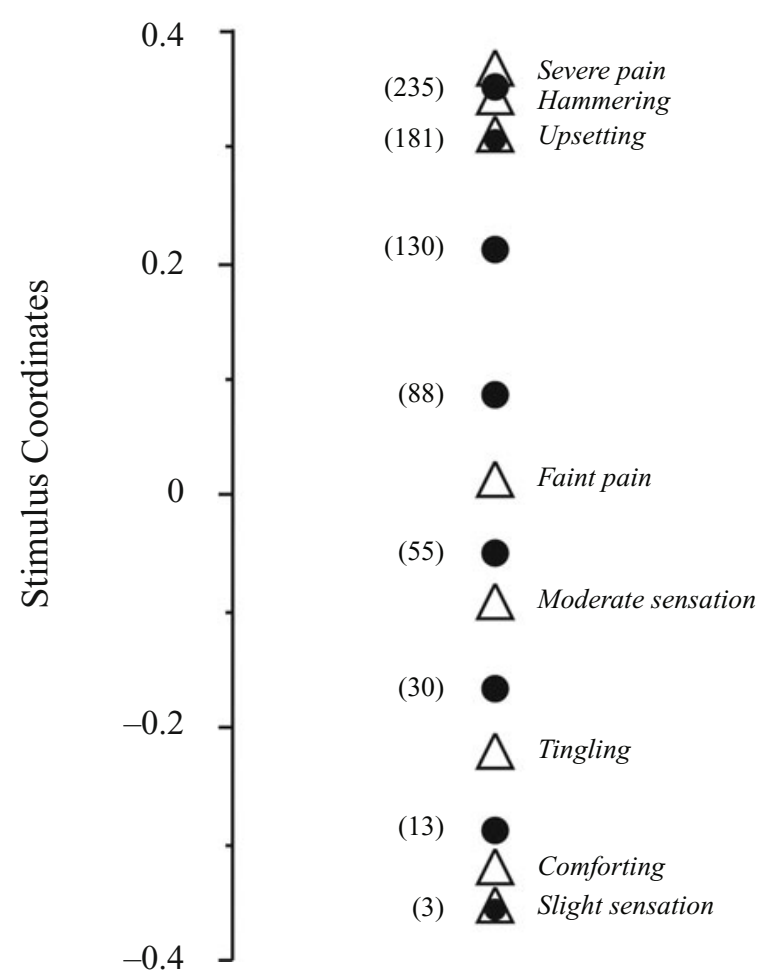

Figure 1. Stimulus coordinates in the group stimulus space obtained by multidimensional individual difference scaling. The onedimensional solution orders the perceived magnitudes expressed as normalized stimulus coordinates for two types of stimulus objects: physical intensities in milliwatts and sensory and emotional descriptors of these experiences. From "Concerning the Homology of Painful Experiences and Pain Descriptors: A Multidimensional Scaling Analysis," by M. N. Janal, 1995, Pain, 64, p. 375. Copyright 1995 by IASP Press. Adapted with permission.

made 120 similarity ratings to all unique pairings of the 16 stimulus objects. The numerical rating scores were entered into half matrices, one for each subject, and analyzed by the SINDSCAL algorithm, an efficient implementation of INDSCAL (Pruzansky, 1975). ${ }^{2}$

Data analysis revealed a one-dimensional solution in the group stimulus space that scaled the stimulus coordinate values of both the electrical stimulus intensities and the sensory and emotion descriptors on a normalized scale in an interdigitated manner from lowest to highest perceived strength (Figure 1). Higher dimensional solutions were viewed but not accepted, because the distributions of the stimulus objects did not yield interpretable dimensions. The results demonstrated the close relationship between the perceived intensities of the sensations induced by the electrical stimuli and the sensory and emotional words that described these experiences.

Method and Results of the Present Study

It was recognized that the stimulus coordinates in the INDSCAL group stimulus space portrayed in Figure 1 


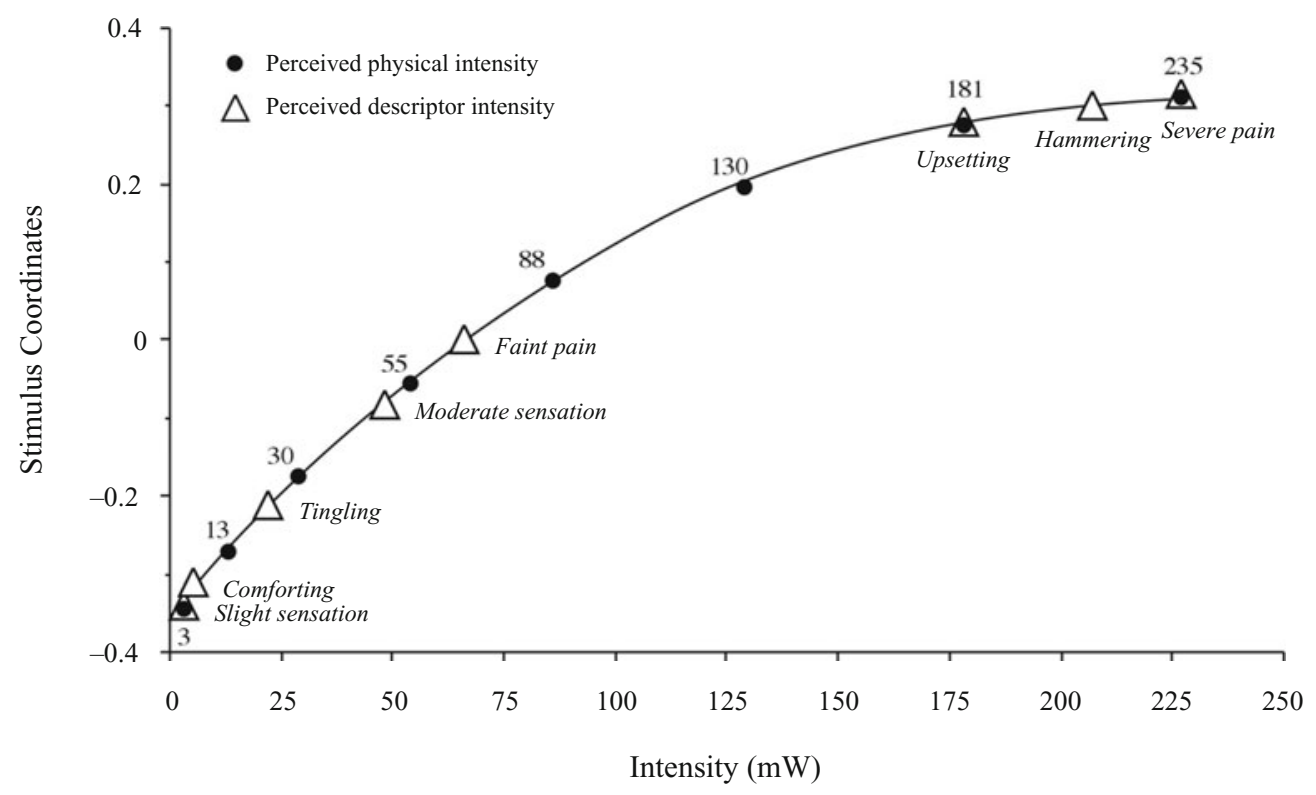

Figure 2. The exponential function obtained by plotting the stimulus coordinate values from Figure 1 (ordinate) against a scale of electrical intensities in milliwatts (abscissa). From "Somatosensory and Pain Measurement by Statistical and Sensory Decision Theory," by W. C. Clark, in Encyclopedia of Neuroscience (3rd ed.), edited by G. Edelman and B. Smith, 2003, Amsterdam: Elsevier. Copyright 2003 by Elsevier. Reprinted with permission.

could be plotted against a scale of electrical intensities in milliwatts. To accomplish this, the stimulus coordinates of the perceived intensity of the electrical stimuli were first plotted (ordinate) against a scale of $0-250 \mathrm{~mW}$ (abscissa). The stimulus coordinates of the descriptors were then projected onto this curve. The result was the exponential function portrayed in Figure 2.
To construct verbal rating scales at the ratio scale level of measurement precision that were arithmetically simple, the stimulus coordinates were arbitrarily rescaled to an origin of zero by adding a constant $(0.4)$ to the stimulus coordinates in Figure 2. In addition, to make the abscissa a general scale applicable to other stimulus modalities, the 0-250 $\mathrm{mW}$ scale was rescaled to $0-100$ Agons $^{3}$ (see Fig-

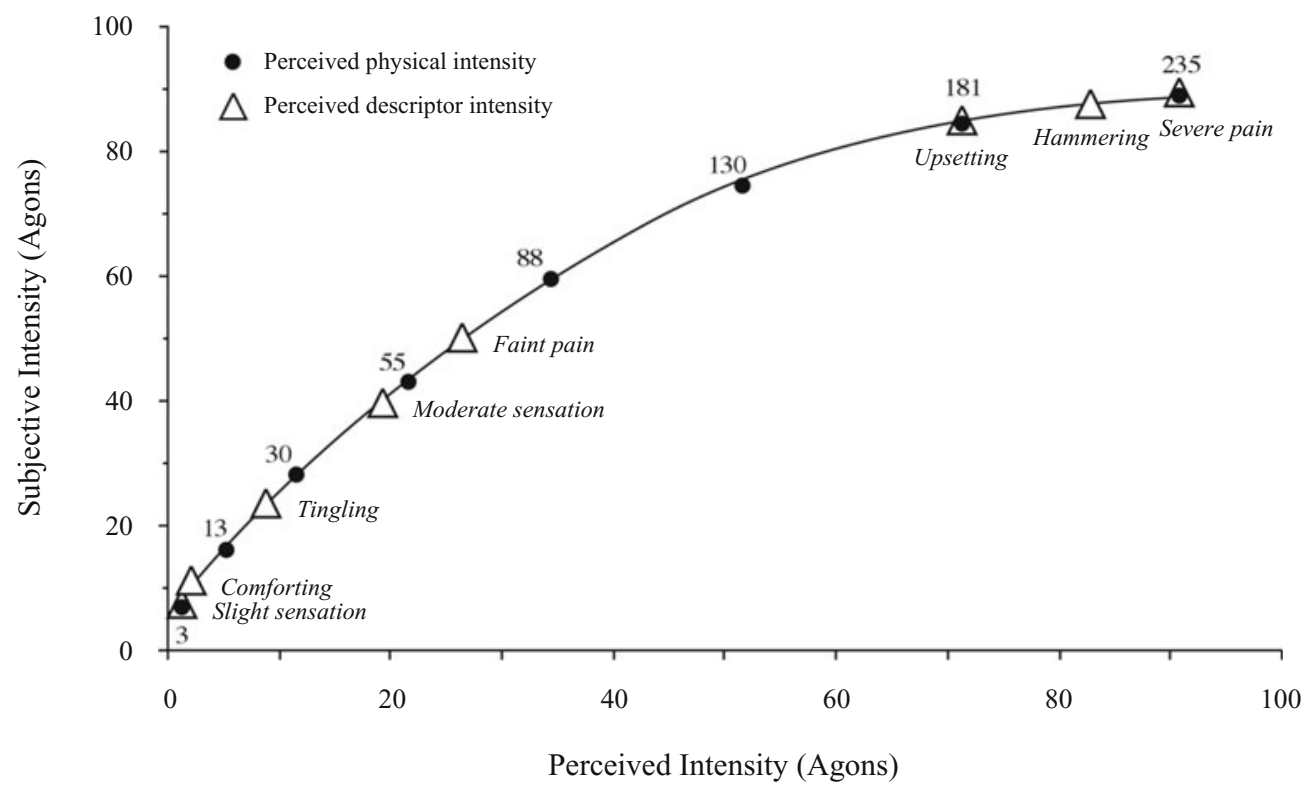

Figure 3. The exponential function obtained when the ordinate is replotted to a subjective intensity scale in Agons with an origin of zero and the abscissa is replotted to a scale of 0 to 100 Agons. 


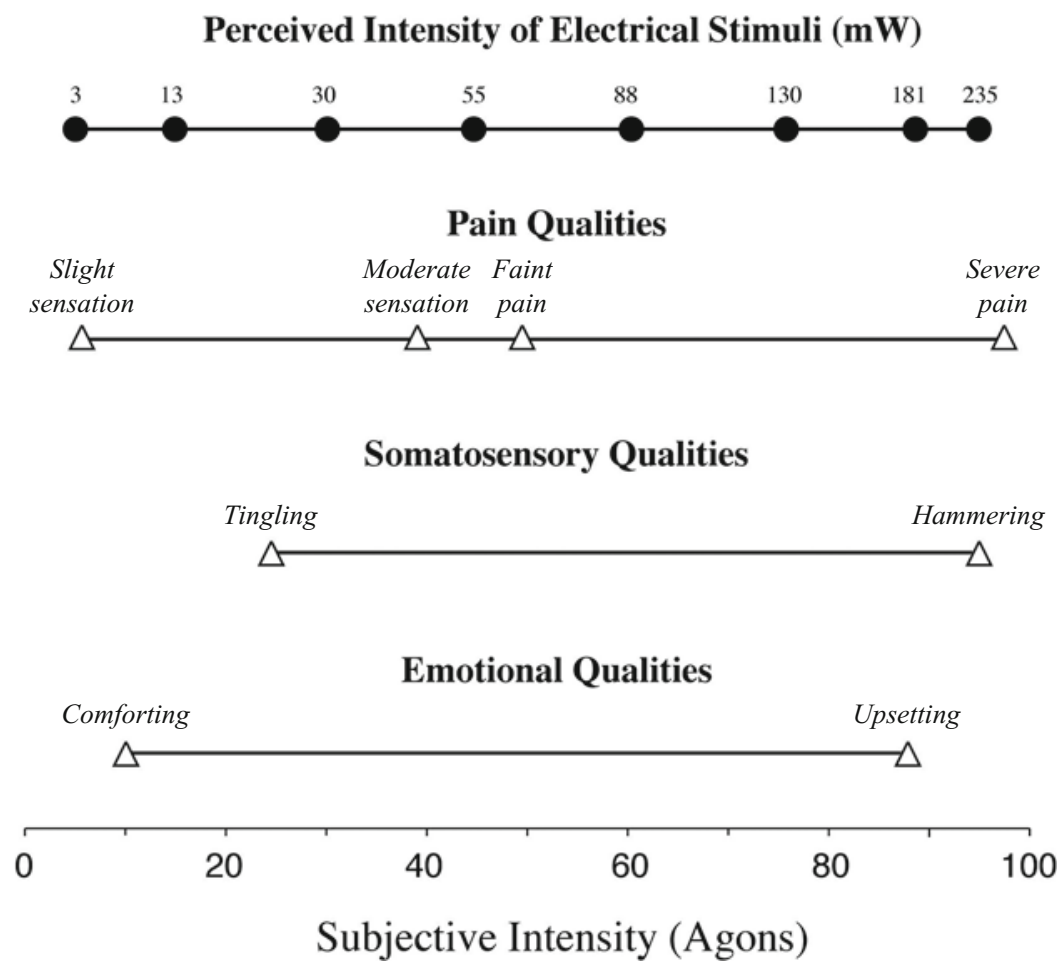

Figure 4. Subjective intensities, measured in Agons, of the perceived intensities of the electrical stimuli $(\mathrm{mW})$ and the descriptors on three verbal scales: pain qualities, somatosensory qualities, and emotional qualities. These scales are at the ratio scale level of measurement precision.

ure 3). Since the milliwatt scale is at the ratio scale level of measurement precision, the Agon scale is also at the ratio scale level of measurement precision.

Four rating scales were constructed from the four attributes that can be distinguished within the perceived intensity dimension (Figure 1). Kruskal and Wish (1978) pointed out that two or more attributes of a dimension may be apparent within a single dimension, but because the attributes share a common underlying dimension (in the present instance, perceived intensity), they do not appear as independent dimensions. (Sometimes the term attribute is used as a synonym for dimension; however, here, we follow the definition given by Kruskal and Wish.) The stimulus coordinates of each of the four attributes - namely, the perceived intensities of the electrical stimuli and the perceived strengths of the eight descriptors in the three verbal categories: pain qualities, somatosensory qualities, and emotional qualities - were plotted as four separate rating scales (Figure 4).

\section{Discussion}

Since the rating scales are at the ratio scale level of measurement precision, the values of the electrical stimuli and descriptors may be read directly from the Agon scale. For example, with respect to the perceived electrical stimulus intensity scale, the subjective strength of the $55-\mathrm{mW}$ stimulus is 44 Agons, and the subjective intensity of the $235-\mathrm{mW}$ stimulus is 95 Agons; accordingly, the perceived intensity of the $235-\mathrm{mW}$ stimulus is 2.2 times that of the $55-\mathrm{mW}$ stimulus. Similarly, for the subjective pain qualities scale, the subjective experience described as severe pain (97 Agons) is 16 times stronger than the subjective experience slight sensation (6 Agons). For the somatosensory qualities scale, the descriptor hammering (93 Agons) is subjectively 4.2 times stronger than tingling (22 Agons); and for the emotional qualities scale, upsetting ( 88 Agons) is perceived as 8.8 times stronger than comforting (10 Agons).

The M-PSP approach is superior to the rating scale techniques currently used because M-PSP allows the legitimate use of parametric statistics. It is unacceptable to use parametric statistics to determine the significance of differences in ordinal scale data, where only the order of the stimulus intensities, not the distance between the stimulus intensities, is known. Stevens (1951) stated that for ordinal scale data, where the basic empirical operation is determination of greater or less, the permissible nonparametric statistical operations are restricted to median, percentiles, and order correlations (type ordinal). However, for ratio scale data, the permissible statistical operations are arithmetic and geometric means, standard deviation, and product-moment correlation.

INDSCAL is the only multidimensional scaling model that, in addition to defining dimensions in the group stimulus space, generates a source or subject coordinate space. The source space quantifies the importance or 
saliency of each dimension to each subject. For a onedimensional solution, such as the one found in the present study, the subject coordinate space quantifies the saliency of each pole - stoical and complainer - to each subject. Unfortunately, because the focus of Janal (1995) was the group stimulus space, the subject coordinate space obtained in that study is no longer available. Instead, we describe the information that the subject coordinate space could have provided. Those subjects who frequently reported the descriptors slight sensation, comforting, and tingling and seldom endorsed severe pain as describing the sensations evoked by stimuli would be located toward the stoical pole, whereas those subjects who frequently reported their sensations as severe pain, hammering, and upsetting would fall toward the complainer pole. Other subjects would be distributed along the dimension between these extremes.

Since the complainer-stoic scale is at the ratio scale level of measurement precision, the subjects' coordinate scores can be treated by parametric statistical techniques and related to scores on other questionnaires, such as the Beck depression scale, or the subjects' coordinate scores can be used to compare different groups of subjects statistically. For example, Clark et al. (1989) compared the coordinate scores of patients suffering cancer-related pain to those of pain-free healthy volunteers. Three dimensions were found in the group stimulus space: pain intensity, sensory qualities, and emotional qualities. Statistical comparison by $t$ test of the subject coordinates demonstrated that the cancer patients found the pain intensity dimension to be the most salient, whereas the healthy volunteers found the emotional qualities dimension to be the most salient.

There are many unresolved questions that the M-PSP procedure can address. For example, is the lower pain threshold of women reported in some, but not all, studies due to a lower (less stoical) pain report criterion, to greater neurosensory sensitivity, or to both? This question could be answered by viewing sex differences in the location of the pain descriptor items and the physical stimulus objects on the exponential function that is depicted in Figure 2 (Clark, 2003a). Comparison of the degree of stoicism between the two groups would yield additional information. The M-PSP procedure could also be used to determine the extent to which the differences in pain thresholds found between anxious and relaxed subjects or among various ethnocultural groups are due to attitudinal differences, to neurosensory sensitivity differences, or to both.

Clearly, the categorical rating scales obtained in this study contain too few items; this is because the M-PSP approach was not envisioned at the time of the earlier study (Janal, 1995). The next study should employ the M-PSP to quantify distances among painful and painless physical stimuli of various intensities and verbal items on a 6-8-point rating scale (e.g., very relaxed, moderately relaxed, slightly relaxed, slightly anxious, moderately anxious, very anxious). Studies using the M-PSP procedure to construct these quantitative scales are now being planned.

\section{Conclusions}

The M-PSP approach to the construction of rating scales at the ratio scale level of measurement precision will greatly improve the quality of the rating scales presented to patients and others. The M-PSP approach has two major advantages over the present procedures for developing rating scales. First, M-PSP rating scales are based on the responses of lay people who will actually be using these scales, not on the judgments of professional caregivers for whom the descriptor may have a different meaning. Second, the ratio scale level of measurement precision permits the legitimate use of parametric statistics to compare different groups of subjects and to compare M-PSP results with scores on standard psychological and physiological tests.

\section{AUTHOR NOTE}

Supported by USPHS Grants NINCDS-20248 and NICDR-12725 and by the Nathaniel Wharton Fund for Research and Teaching in Brain, Body and Behavior. Parts of this study were presented at the European Chapter of the International Association for the Study of Pain, Prague, Czech Republic, 2003, and at the International Association for the Study of Pain, Sydney, Australia, 2005. Correspondence concerning this article should be addressed to W. C. Clark, Department of Psychiatry, Box 50, Columbia University, 1051 Riverside Dr., New York, NY 10032-2695 (e-mail: clarkcr@pi.cpmc.columbia.edu).

\section{REFERENCES}

BAIRD, J. C., \& Noma, E. (1978). Fundamentals of scaling and psychophysics. New York: Wiley.

BERNOULLI, D. (1954). Exposition of a new theory on the measurement of risk (L. Sommer, Trans.). Econometrica, 22, 23-36. (Original work published 1738)

Carroll, J. D., \& Chang, J.-J. (1970). Analysis of individual differences in multidimensional scaling via an $N$-way generalization of "Eckart-Young" decomposition. Psychometrika, 35, 283-319.

CLARK, W. C. (2003a, Sept.). Do women actually experience more pain than men or do they merely report more? A multidimensional scaling approach. Poster presented at the 4th Congress of the European Federation of International Association for the Study of Pain Chapters (EFIC), Prague.

ClARK, W. C. (2003b). Pain, emotion and drug induced subjective states: Applications of multivariate scaling. In G. Adelman \& B. Smith (Eds.), Encyclopedia of neuroscience (3rd ed.). Amsterdam: Elsevier.

Clark, W. C. (2003c). Somatosensory and pain measurement by statistical and sensory decision theory. In G. Adelman \& B. Smith (Eds.), Encyclopedia of neuroscience (3rd ed.). Amsterdam: Elsevier.

Clark, W. C., Carroll, J. D., Yang, J. C., \& Janal, M. N. (1986). Multidimensional scaling reveals two dimensions of thermal pain. Journal of Experimental Psychology: Human Perception \& Performance, 12, 103-107.

Clark, W. C., Ferrer-Brechner, T., Janal, M. N., Carroll, J. D., \& YANG, J. C. (1989). The dimensions of pain: A multidimensional scaling comparison of cancer patients and healthy volunteers. Pain, 37, 23-32.

Clark, W. C., Janal, M. N., Hoben, E. K., \& Carroll, J. D. (2001). How separate are the sensory, emotional, and motivational dimensions of pain? A multidimensional scaling analysis. Somatosensory \& Motor Research, 8, 31-39.

FeCHNer, G. T. (1966). Elements of psychophysics (Vol. 1, H. E. Adler, Trans.) New York: Holt, Rinehart \& Winston. (Original work published 1860)

Gracely, R. H., McGrath, P., \& Dubner, R. (1978). Ratio scales of sensory and affective verbal pain descriptors. Pain, 5, 5-18.

JANAL, M. N. (1995). Concerning the homology of painful experiences and pain descriptors: A multidimensional scaling analysis. Pain, 64, 373-378.

Janal, M. N., Clark, W. C., \& Carroll, J. D. (1993). Multidimensional scaling of painful electrocutaneous stimulation: INDSCAL di- 
mensions, signal detection theory indices, and the McGill Pain Questionnaire. Somatosensory \& Motor Research, 10, 31-39.

KRUSKAL, J. B., \& WiSh, M. (1978). Multidimensional scaling. Beverly Hills: Sage.

Lattin, J. M., Carroll, J. D., \& Green, P. E. (2003). Analyzing multivariate data. Belmont, CA: Duxbury Press.

PRUZANSKY, S. (1975). How to use SINDSCAL: A computer program for individual differences in multidimensional scaling. Murray Hill, NJ: ATT \& Bell Laboratories.

Stevens, S. S. (1951). Mathematics of measurement and psychophysics. In S. S. Stevens (Ed.), Handbook of experimental psychology. New York: Wiley.

Stevens, S. S. (1957). Concerning the form of the loudness functions. Journal of the Acoustical Society of America, 29, 603-606.

\section{NOTES}

1. Women were excluded because we planned to use the same subjects in a subsequent study of the effect of an analgesic on the dimensional structure of the group stimulus space, and we were concerned that the local institutional review board might not approve the inclusion of women in a drug study.

2. The SINDSCAL program is available on a diskette provided with Lattin et al. (2003).

3. Agon is from the Greek word meaning "an agonizing physical contest."

(Manuscript received October 7, 2008;

revision accepted for publication September 10, 2009.) 